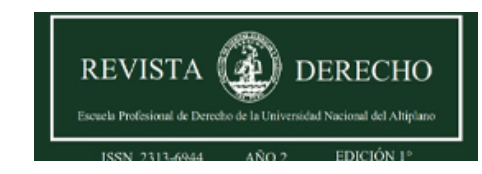

Revista de Derecho

ISSN: 2313-6944

ISSN: 2707-9651

revistaderecho@unap.edu.pe

Universidad Nacional del Altiplano

Perú

\title{
El test de igualdad según la jurisprudencia del Tribunal Constitucional: reflexiones sobre la sentencia recaída en el Expediente N. ${ }^{\circ} 00045-2004-\mathrm{PI} / \mathrm{TC}$ y 00004-2006-PI/TC
}

Muñoz Valeriano, William J.

El test de igualdad según la jurisprudencia del Tribunal Constitucional: reflexiones sobre la sentencia recaída en el Expediente N. ${ }^{\circ} 00045-2004-\mathrm{PI} / \mathrm{TC}$ y 00004-2006-PI/TC

Revista de Derecho, vol. 5, núm. 2, 2020

Universidad Nacional del Altiplano, Perú

Disponible en: https://www.redalyc.org/articulo.oa?id=671870937005

DOI: https://doi.org/10.47712/rd.2020.v5i2.92

\section{(c) (1)}

Esta obra está bajo una Licencia Creative Commons Atribución 4.0 Internacional. 


\title{
El test de igualdad según la jurisprudencia del Tribunal Constitucional: reflexiones
} sobre la sentencia recaída en el Expediente N. ${ }^{\circ}$ 00045-2004-PI/TC y 00004-2006$\mathrm{PI} / \mathrm{TC}$

The equality test according to the jurisprudence of the Constitutional Court: reflections on the sentence passed in File No. 00045-2004-PI / TC and 00004-2006-PI / TC

William J. Muñoz Valeriano

Asesor Legal en la UNAP, Perú

DOI: https://doi.org/10.47712/rd.2020.v5i2.92

Redalyc: https://www.redalyc.org/articulo.oa?

id $=671870937005$

Recepción: 30 Septiembre 2020

Aprobación: 25 Octubre 2020

Publicación: 27 Octubre 2020

\begin{abstract}
:
El objetivo del trabajo es analizar y dar cuenta de los aspectos esenciales que encierra el test de igualdad desde la perspectiva de la jurisprudencia constitucional, ya que en los últimos años dicho test fue utilizado por diversas instancias judiciales, sin embargo, su uso se dio de forma inadecuada porque muchas veces su aplicación no se orientó por lo desarrollado en el Expediente $\mathrm{N}^{\circ}$ 00045-2004-PI/TC y 00004-2006-PI/TC, es más, ni el mismo órgano emisor lo sigue, siendo así, la tesis central que se asume aquí es que los criterios desarrollados por el Tribunal Constitucional en el Expediente N. ${ }^{\circ}$ 00045-2004-PI/TC y 00004-2006$\mathrm{PI} / \mathrm{TC}$-sobre el test de igualdad- sean aplicados de manera uniforme, de esa manera sería mucho más sencillo establecer si un hecho o acto constituye tratamiento justificado o injustificado, además, se evitan las inconsistencias en su aplicación; por último, se propone que lo recogido en esa sentencia sea declarada precedente constitucional para que genere predictibilidad la aplicación del mencionado test.
\end{abstract}

KEYWORDS: Constitution, right to equality, fundamental rights, equality test.

\section{Abstract:}

The objective of the work is to analyze and account for the essential aspects contained in the equality test from the perspective of constitutional jurisprudence, since in recent years this test was used by various judicial instances, however, its use was given inadequate way because many times its application was not guided by what was developed in File No. 00045-2004-PI / TC and 00004-2006-PI / TC, moreover, not even the issuing body itself follows it, being so, the The central thesis that is assumed here is that the criteria developed by the Constitutional Court in File No. 00045-2004-PI / TC and 00004-2006-PI / TC -on the equality test- be applied in a uniform manner, In this way, it would be much easier to establish whether a fact or act constitutes justified or unjustified treatment, in addition, inconsistencies in its application are avoided; Finally, it is proposed that what is contained in that judgment be declared a constitutional precedent so that the application of the aforementioned test generates predictability.

KEYWORDS: Constitution, right to equality, fundamental rights, equality test.

PalabRas ClaVE: Constitución, derecho a la igualdad, derechos fundamentales, test de igualdad

\section{INTRODUCCIÓN}

El lema bajo la cual la Revolución Francesa del año de 1789 se proclamó fue: libertad, igualdad y fraternidad; la misión de las generaciones posteriores debía ser concretizar estos mandatos. En el devenir de la historia la libertad, igualdad y fraternidad se desarrollaron profusamente, pese a esto, casi todas las cartas constitucionales tienen una remisión y referencia a estos tres aspectos, ya que el constitucionalismo contemporáneo ha incorporado dentro de su discurso el derecho a la igualdad, la libertad y la solidaridad (una forma de manifestación de la fraternidad); por eso, hoy en día, las cartas constitucionales reconocen 
estos tres derechos[1] (Mosquera, 2010; Pérez, 2005). Los mismos que son fundamentales para comprender la dinámica del constitucionalismo contemporáneo.

También hay que recordar que es importante el conocimiento de la jurisprudencia del Tribunal Constitucional porque permite entender mejor los alcances de un derecho y el significado de protegerlo, además, esos desarrollos son válidos para pedir su protección frente a la vulneración o restricción, si se desconoce sobre los alcances de los derechos y lo que ha dicho el máximo intérprete de la Constitución, es poco probable que pueda aspirarse a una protección óptima y válida del mismo. Una de las consideraciones notables es conocer la jurisprudencia constitucional para proteger mejor un derecho. En tal sentido, si bien es cierto que dentro del constitucionalismo el reconocimiento de derechos en las constituciones son elementos importantes, también se ha sumado a ello la actividad jurisprudencial del Tribunal Constitucional que tiene como finalidad dar mayores alcances sobre el funcionamiento y el propósito de los derechos fundamentales, por esa razón en este trabajo dedicamos especial atención al desarrollo jurisprudencial sobre el test de igualdad.

En todo esto, un aspecto que sale a la palestra de la discusión consiste en preguntas sobre qué es el test de igualdad, para qué sirve, cuáles son los ámbitos de aplicación y cómo el Tribunal Constitucional ha venido dándole contenido al mismo. También se desarrolla algunos aspectos generales sobre el derecho a la igualdad y su posición en el sistema constitucional peruano, además, conocer sobre los problemas relacionados la aplicación del derecho a la igualdad. Teniendo en cuenta que dicho derecho es de carácter fundamental, pero sus alcances o dimensiones son difíciles determinar porque es ambiguo y vago, a pesar de eso el Tribunal Constitucional ha sabido darle contenido para que sea aplicable a diversas circunstancias donde se invoca la vulneración de dicho derecho, por eso, es importante efectuar una referencia sumaria y sucinta del mismo.

La revisión preliminar del desarrollo de la jurisprudencia del Tribunal Constitucional exhibe un problema que consiste en el apartamiento de los pasos de verificación del test de igualdad, ya que en los Expedientes N. 00045-2004-PI/TC y 00004-2006-PI/TC se fijó que la aplicación del test debe seguir estos pasos: (a) primer paso (verificación de la diferenciación legislativa), (b) segundo paso (determinación de la intensidad de la intervención en la igualdad, (c) tercer paso (verificación de la existencia de un fin constitucional en la diferenciación), (d) cuarto paso (examen de idoneidad) y (e) quinto paso (examen de necesidad). Luego, se advierte que en sentencias como Exp. $\mathrm{N}^{\circ}$ 0020-2017-PA/TC, Exp. $\mathrm{N}^{\circ}$ 09833-2016-PA/TC o Exp. N -02345-2009-PA/TC, el mismo Tribunal Constitucional no utiliza o sigue los mismos pasos, sino que se limita a invocar el término de comparación válida, la utilización de tres pasos o la invocación directa de los pasos del test de proporcionalidad (idoneidad, necesidad y proporcionalidad en sentido estricto). Estos aspectos resultan complejos de comprender y conocer con claridad los pasos que deben seguirse.

En el trabajo se pone especial énfasis en el derecho a la igualdad y, en específico, el test de igualdad. En ese sentido, en el desarrollo del trabajo abordamos los siguientes ámbitos: a) explicar el derecho a la igualdad y sus manifestaciones a través del desarrollo jurisprudencial del Tribunal Constitucional; b) resaltar los contenidos o alcances del test de igualdad y c) desarrollar el test de igualdad según el Tribunal Constitucional, de acuerdo al desarrollo jurisprudencial, considerando que no se tomarán todas las decisiones, sino los más importantes y relevantes. También se echará mano de algunas precisiones sobre el derecho a la igualdad y el test de igualdad con la ayuda de la doctrina nacional e internacional. Todo eso para concluir que la jurisprudencia del Tribunal Constitucional, en especial, al momento de aplicar el test de igualdad debe guiarse por las pautas fijadas en los Expedientes N. ${ }^{\circ} 00045-2004-P I / T C$ y 00004-2006-PI/TC.

Finalmente, queda resaltar que este trabajo tiene como propósito analizar que la aplicación del test de igualdad se oriente por lo establecido en el Exp. N. ${ }^{\circ}$ 00045-2004-PI/TC y 00004-2006-PI/TC, en especial, en lo relacionado con los pasos o estructura del test, ya que muchas veces resulta complicado determinar cuándo una situación viola o contraviene la igualdad, siendo así, los pasos desarrollados por el máximo órgano de interpretación de la Constitución sobre el test de igualdad para verificar si la diferenciación introducida por las normas y los actos cuestionados son válidas o constituyen una discriminación, resultan esenciales. 


\section{LAS CONSIDERACIONES SOBRE EL DERECHO A LA IGUALDAD EN LA JURISPRUDENCIA DEL Tribunal Constitucional}

El Tribunal Constitucional es el supremo intérprete de la Constitución, es decir, es quién tiene la última palabra en materia de interpretación constitucional, tal como lo reconoce la Ley Orgánica del Tribunal Constitucional en su artículo primero, ya señala lo siguiente: "El Tribunal Constitucional es el órgano supremo de interpretación, integración y control de la constitucionalidad", por este motivo, es menester acudir a las elucidaciones que ha efectuado sobre el derecho a la igualdad. En esa línea, en el presente acápite, esencialmente se tocará los siguientes aspectos desarrollados por la jurisprudencia del Tribunal Constitucional sobre el derecho a la igualdad: a) contenido, b) concepto, y, c) dimensiones. Es importante considerar esos puntos porque permiten conocer sobre la relevancia del derecho a la igualdad y la forma de protección en sede constitucional.

Es oportuno mencionar que, en términos del Tribunal Constitucional, la "noción de igualdad debe ser percibida en dos planos convergentes. En el primero aparece como un principio rector de la organización y actuación del Estado Democrático de Derecho. En el segundo, se presenta como un derecho fundamental de la persona"; en ese escenario, ha ampliado considerando que como "principio implica un postulado o proposición con sentido y proyección normativa o deontológico, que, por tal, constituye parte del núcleo del sistema constitucional de fundamento democrático. Como derecho fundamental comporta el reconocimiento de la existencia de una facultad o atribución conformante del patrimonio jurídico de una persona, derivada de su naturaleza, que consiste en ser tratada igual que los demás en relación a hechos, situaciones o acontecimientos coincidentes; por ende, como tal deviene en el derecho subjetivo de obtener un trato igual y de evitar los privilegios y las desigualdades arbitrarias" (Ferrajoli, 2010).

Una vez expuesto las consideraciones previas, el Supremo Intérprete de la Constitución, conceptualiza la igualdad como "un - principio- derecho que instala a las personas situadas en idéntica condición, en un plano de equivalencia. Ello involucra una conformidad o identidad por coincidencia de naturaleza, circunstancia, calidad, cantidad o forma, de modo tal que no se establezcan excepciones o privilegios que excluyan a una persona de los derechos que se conceden a otra, en paridad sincrónica o por concurrencia de razones" (Exp. $\mathrm{N}^{\circ}$ 0261-2003-AA/TC fundamento jurídico 3). En la misma línea, el Tribunal Constitucional, ha insistido que la igualdad es "un principio-derecho que instala a las personas, situadas en idéntica condición, en un plano de equivalencia. Ello involucra una conformidad o identidad por coincidencia de naturaleza, circunstancia, calidad, cantidad o forma, de modo tal que no se establezcan excepciones o privilegios que excluyan a una persona de los derechos que se conceden a otra, en paridad sincrónica o por concurrencia de razones" (Eguiguren, 2006; Eto, 2016; Exp. №3533-2003-AA/TC fundamento jurídico 4) [2].

Para cerrar este punto, además, es oportuno considerar que el derecho fundamental a la igualdad es un principio-derecho que ubica a todas las apersonas en las mismas condiciones y prerrogativas, por ende, involucra una conformidad o identidad por coincidencia de naturaleza, circunstancia, calidad, cantidad o forma, de modo tal que no se establezcan excepciones o privilegios que excluyan a una persona de los derechos que se conceden a otra, en paridad sincrónica o por concurrencia de razones. Eso es igualdad en términos generales, siendo así, podemos notar que es importante su protección en todas las esferas y dimensiones para que no se produzcan diferenciaciones o desequiparaciones indebidas.

2.1. El derecho a la igualdad y el test de igualdad en la jurisprudencia del Tribunal Constitucional:

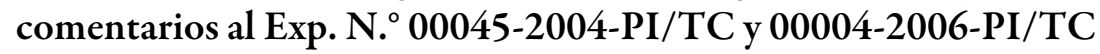

Es conocido que se ha desarrollado el test de igualdad en la jurisprudencia peruana, por primera vez, en la sentencia del Tribunal Constitucional -Exp. N. ${ }^{\circ}$ 00045-2004-PI/TC y 00004-2006-PI/TC-, por ende, requiere análisis de su contenido para conocer sobre los alcances del mismo y la forma de emplearlo en los futuros casos. El principal problema con ese tema es que la sentencia carece de calidad de precedente vinculante, por ende, notaremos que el empleo del test de igualdad para solucionar situaciones concretas no 
se visualiza de forma adecuada, esto es, se aplica de forma difusa y variada, situación que impide conocer la forma de aplicación del mismo, en ese contexto, lo que hacemos aquí son algunos alcances concretos para que se apliquen los pasos del test de igualdad según lo definido por esta sentencia y, además, fijar una definición.

Habiendo hecho esas advertencias, ahora, debemos anotar que el Tribunal Constitucional y cualquier órgano de administración de justicia, al momento de aplicar el test de igualdad, obligatoriamente debe utilizar los pasos diseñados en los expedientes Expedientes N. ${ }^{\circ} 00045-2004-\mathrm{PI} / \mathrm{TC}$ y 00004-2006-PI/TC porque es más integral y compacto para comprender los alcances del test. En ese sentido, a raíz de la revisión preliminar del contenido de algunas decisiones donde se advierte que no se siguen los lineamientos fijados en las sentencias de los expedientes mencionados, lo que se propone es que el desarrollo del test de igualdad debe guardar coherencia con lo recogido en las sentencias antes citadas. Y es que no puede considerarse que los pasos diseñados en la sentencias no sean trabajados correcta y adecuadamente por los órganos de administración de justicia constitucional.

En suma, si bien es cierto que la línea jurisprudencia del Tribunal Constitucional sobre el derecho a la igualdad se mantenido durante los años, considerando que la igualdad es un principio-derecho, el mismo que no ha cambiado hasta la actualidad. Teniendo esa base, además, es oportuno considerar que el test de igualdad busca solucionar problemas vinculados con el tratamiento desigual y desequiparación de condiciones, de tal modo que se evite la discriminación o diferenciación irrazonable y arbitraria. Ergo, el gran inconveniente es que el razonamiento elaborado por diversos órganos judiciales y el mismo Tribunal Constitucional es que no puede garantizar la aplicación uniforme y correcta del test de igualdad -de acuerdo a la sentencia referidaporque se usa de forma difusa e inconsistente, por ende, eso no permite ver adecuadamente el razonamiento jurídico de las demás instancias judiciales con relación a su aplicación.

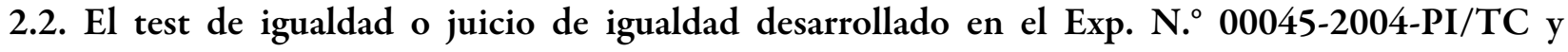
00004-2006-PI/TC: una crítica desde la posición personal

El jurista colombiano Carlos Bernal afirma que este este test o juicio de igualdad a estado expuesto a versiones distintas, entre las que podemos mencionar son: a) juicio de igualdad de influencia europea, que sigue el modelo del principio de proporcionalidad, b) juicio de influencia norteamericana, que se funda en la distinción entre tres tipos de escrutinios de igualdad, y, c) juicio integrado de igualdad que pretende ser una simbiosis entre los dos primeros esquemas (Cfr. Bernal, 2008, p. 262). El Tribunal Constitucional peruano ha utilizado este test para determinar cuándo se produce trato desigual objetivo y cuando es arbitrario, en ese sentido, ha explicado que este test consiste en "determinar cuándo estamos frente a un trato desigual con base en justificaciones objetivas y razonables; o cuándo frente a un trato arbitrario, caprichoso o injustificado y, por tanto, discriminatorio" (Exp. N $\mathrm{N}^{\circ}$ 02776-2011-PHD/TC fundamento jurídico 11).

El Tribunal Constitucional para aplicar el test de igualdad, esto con la finalidad de verificar si la diferenciación introducida por las normas y los actos cuestionados son válidas o constituyen una discriminación, ha elaborado un juicio o test de igualdad, el mismo que recoge un conjunto de pasos, que a continuación pasamos a exponer: a) El primer paso consiste en la verificación de la existencia de un fin constitucional en la diferenciación, lo cual implica que "la diferenciación efectuada por el legislador no vulnere el principio-derecho de igualdad" (Exp. N 0004-2006-PI/TC fundamento jurídico 138); b) El segundo paso es el juicio de proporcionalidad, el mismo que se debe tomar en consideración si la medida dictada resulta proporcional con el fin que se pretende obtener; c) El examen de idoneidad, este paso exige que la medida legislativa que establece la diferencia de trato deba ser congruente con el fin legítimo que se trata de proteger. En otras palabras, se evalúa si la medida legislativa es idónea para conseguir el fin pretendido por el legislador. Por el contrario, si se verifica que la medida adoptada por el legislador no guarda ninguna relación con el fin que se trata de proteger, esta limitación resultará inconstitucional; d) el examen de necesidad, en este paso, se exige que la medida adoptada por el legislador, para ser constitucional, deba ser absolutamente indispensable para la consecución del fin legítimo, pues de existir una medida alternativa que, siendo igualmente idónea para conseguir el mencionado fin, influya con menor intensidad en el respectivo 
bien constitucional, entonces la medida legislativa cuestionada resultará inconstitucional, y, e) el examen de proporcionalidad en sentido estricto, este comprende la comparación entre dos pesos o intensidades (Alvites, 2006): a) aquel que se encuentra en la realización del fin de la medida legislativa diferenciadora; y, b) aquel que radica en la afectación del derecho fundamental de que se trate, de manera tal que la primera de estas deba ser, como se ha mencionado, por lo menos, equivalente a la segunda[3].

En la doctrina nacional se advierte que "el Tribunal ha desarrollado dos versiones del test: uno de igualdad, cuando se discute sobre este derecho específico, que consta de los seis pasos que establece la sentencia 0045-2004-PI/TC, y otro de cinco pasos para la discusión de los demás derechos [...]. Sin embargo, el Tribunal usa indistintamente los pasos del test: a veces usa los seis, otras veces los cuatro últimos, otras veces hace combinaciones variadas de los diferentes pasos" (Rubio, 2011, p. 24). Esto muestra que la aplicación del test es variable, pero el objetivo de limitar las actuaciones y las normas que intervienen el derecho a la igualdad se mantienen.

Finalmente, si bien es cierto que el Tribunal Constitucional ha desarrollado la importancia del test de igualdad, sin embargo, todavía queda como una tarea pendiente solucionar asuntos vinculados con su aplicación correcta, ya que muchos órganos judiciales no lo aplican de forma adecuada, incluso, el mismo Tribunal no sigue sus elaboraciones previas. La crítica radica en que no ha fijado como precedente el Tribunal Constitucional la forma de aplicación del test de igualdad, por ende, eso genera diversos problemas prácticos de aplicación concreta. También ese problema se puede solucionar legislando el tema, es decir, garantizando la aplicación concreta y correcta del asunto a otros casos o supuestos mediante una ley, así evitar razonamientos dispersos sobre la aplicación del test de igualdad.

\section{3. ¿Cuál es la importancia de establecer el concepto del test de igualdad?}

Habiendo mencionado que el test de igualdad debe guiarse por lo fijado en el Exp. N. ${ }^{\circ} 00045-2004-P I / T C$ y 00004-2006-PI/TC por el Tribunal Constitucional, siendo así, ahora se debe establecer la importancia del mismo, esto es, si tiene algún propósito concreto y los fines prácticos que tiene el test de igualdad. Dilucidar ese asunto es importante porque permite explicar con mayores detalles sobre las dimensiones de su aplicación al momento de aplicar a un caso concreto, mientras solamente se indique solamente los pasos o etapas de aplicación, entonces es posible que no se entienda muy bien sobre los ámbitos de su uso e importancia. El asunto problemático es que la definición del test de igualdad no fue establecido por la sentencia que es materia de comentario, esa es la razón que motiva a que se fije el mismo para que sea más claro y de esa manera conozcan los operadores jurídicos sobre los ámbitos o dimensiones de aplicación del mismo

Expediente $\mathrm{N}^{\circ}$ 0001-2004-PI; Expediente STC 0019-2010-PI; Expediente $\mathrm{N}^{\circ}$ 0045-2004-PI y Expediente Exp. $\mathrm{N}^{\circ}$ 0261-2003-AA/TC, puntualmente, ha quedado claro que el test tiene diversas vertientes o formas de aplicarse. Eso ha suscitado que se busque la aplicación concreta y correcta del test, en especial, siguiendo el esquema trazado en el Exp. 00045-2004-PI/TC. Teniendo eso como base, lo concreto es que tampoco se puede advertir de dicha sentencia que exista una definición clara y prístina sobre el mismo, siendo así, lo más importante es que el test sea visto como una herramienta para la solución de situaciones que impliquen problemática de derechos fundamentales, en especial, el derecho a la igualdad. Lo que se desprende de eso es que el test podrá se aplicado en términos concretos y correctos, sin que existan problemas en su concepción, ya que es otro inconveniente que genera la dispersión de aplicación o uso del mismo.

En suma, con todo lo indicado queda claro el test de igualdad es una herramienta que sirve para garantizar el derecho a la igualdad y sirve para determinar la vulneración de dicho derecho cuando se producen hechos de desigualdad. Eso refleja que es importante para determinar cuando una situación o hecho concreto genera desigualdad, ya que la finalidad es proteger el derecho fundamental a la igualdad, sin un instrumento con el test de igualdad, no se podría identificar las circunstancias que desembocan en trato desigual e injustificado. También vemos pertinente que definir el test de igualdad sirve para conocer la importancia de su aplicación al momento de solucionar problemas vinculados con el trato desigual en que pudiesen incurrir tanto el poder público o privado. 


\section{CONCLUSIÓN}

El test de igualdad desarrollado por el Tribunal Constitucional en el Exp. N. ${ }^{\circ}$ 00045-2004-PI/TC y 00004-2006-PI/TC sirve para orientar su uso en otras decisiones, ya que permite identificar adecuadamente sobre la importancia que tiene el mismo y establece pasos definidos que se deben seguir para identificar si un hecho o acto constituye tratamiento justificado o injustificado, eso por un lado es bueno; sin embargo, por otro lado, advertimos que el mismo Tribunal Constitucional, en esa sentencia y en otras, no ha precisado sobre la importancia del test así como tampoco sobre los pasos definitivos que deben utilizarse, siendo así, creemos que debe mantenerse la posición asumida en el Exp. N. ${ }^{\circ}$ 00045-2004-PI/TC y 00004-2006-PI/TC porque ya existe seguridad y uniformidad sobre el mismo, eso en cuanto a los pasos para aplicar el test de igualdad. Ahora, con relación a la definición o finalidad del mismo es que sirve para identificar situaciones que suponen trato injustificado o arbitrario, por ende, es una herramienta que pueden emplear los operadores jurídicos para dilucidar aquellas situaciones, hechos o normas que introducen tratos desigualitarios.

\section{BIBLIOGRAFÍA}

Alvites, E. (2006). "Igualdad y derechos sociales. Reflexiones”. S. Mosquera (Coord.), El derecho fundamental de igualdad (pp. 140 - 160). Lima, Perú: Palestra Editores.

Eguiguren Praeli, F. (2006). Estudios Constitucionales. Lima, Perú: ARA Editores

Eto Cruz, G. (2016). Constitución Peruana. Historia y Dogmática. Lima, Perú: Editorial Adrus.

Ferrajoli, L. (2010). Derechos y Garantías. La ley del más débil. Madrid, Lima: Editorial Trotta.

Mosquera Monelos, S. (2010) "El derecho a la igualdad y la no discriminación por razón de religión” En S. Mosquera (Coord.). El derecho fundamental de igualdad (pp. 14 - 30). Lima: Palestra Editores.

Pérez Luño, A. (2005) "Igualdad". En 10 palabras clave sobre derechos humanos. Navarra, España: Editorial Verbo Divino.

Rubio Correa, M. (2011). El test de proporcionalidad en la jurisprudencia del Tribunal Constitucional peruano. Lima, Perú: Pontificia Universidad Católica del Perú.

Sentencia Tribunal Constitucional. Expediente N ${ }^{\circ}$ 0001-2004-PI. Fecha: 22 de enero de 2005. (visitado el 01 de agosto de 2017: www.tc.gob.pe/jurisprudencia)

Sentencia Tribunal Constitucional. Expediente STC 0019-2010-PI. Fecha: 20 de febrero de 2013. (visitado el 01 de agosto de 2017: www.tc.gob.pe/jurisprudencia)

Sentencia Tribunal Constitucional. Expediente $N^{\circ}$ 0045-2004-PI. Fecha: 18 de mayo de 2006. (visitado el 01 de agosto de 2017: www.tc.gob.pe/jurisprudencia)

Sentencia Tribunal Constitucional. Expediente Exp. N 0261-2003-AA/TC. Fecha: 20 de setiembre de 2005. (visitado el 01 de agosto de 2017: www.tc.gob.pe/jurisprudencia)

\section{Notas}

[1] En el Perú la Constitución Política de 1993, en sus disposiciones constitucionales, ha regulado de forma explícita el derecho a la igualdad (artículo $2^{\circ}$ ), la libertad (artículo $2^{\circ}$ ) y la fraternidad (artículo $14^{\circ}$ ).

[2] Este Tribunal, en reiteradas sentencias como las recaídas en el Exp. N. ${ }^{\circ}$ 0261-2003-AA/TC, Exp. N 010-2002-AI/ TC, Exps. Acumulados Nos 0001/0003-2003-AI/TC), Exp. No 0261-2003-AA/TC, entre otros, has sostenido que la igualdad es un principio-derecho que ubica a las personas en las mismas condiciones. En otra decisión, ha puesto de manifiesto que “(...) el principio de igualdad no se encuentra reñido con el reconocimiento legal de la diferencia de trato, en tanto ésta se sustente en una base objetiva, razonable, racional y proporcional. El tratamiento jurídico de las personas debe ser igual, salvo en lo atinente a la diferencia de sus 'calidades accidentales' y a la naturaleza de las cosas que las vinculan coexistencialmente" (Exp. 0018-2003-AI). Adicionalmente, debe tenerse en cuenta que existe vinculación negativa y positiva a la igualdad: "La vinculación negativa se traduce en la exigencia de tratar igual a los que son "iguales" 
William J. Muñoz Valeriano. El test de igualdad según la jurisprudencia del Tribunal Constituciona...

y "distinto" a los que son distintos, de forma tal que la ley, como regla general, tenga una vocación necesaria por la generalidad y la abstracción, quedando proscrita la posibilidad que el Estado, a través del legislador, pueda ser generador de factores discriminatorios de cualquier índole. La vinculación positiva supone la exigencia de revertir las condiciones de desigualdad o de reponer las condiciones de igualdad de las que la realidad social pudiera estarse desvinculando, en desmedro de las aspiraciones constitucionales, a través de las acciones positivas cuyo fin sea promover real y efectivamente la igualdad sustancial entre los individuos. Ello a efectos de configurar materialmente una simetría de oportunidades para todos los seres humanos. En ese sentido, no se considera como discriminaciones aquellas acciones legislativas que establezcan tratos diferenciados con el objeto de promover la igualdad real de oportunidades, a condición de que dicha acción afirmativa esté sujeta a la regla de temporalidad" (Exp. Nº18-2003-AI/TC fundamento jurídico 5).

[3] Es recomendable remitir a la siguiente sentencia: Exp. $\mathrm{N}^{\circ}$ 00004-2006-PI/TC fundamentos jurídicos 138 - 147 , asimismo, en la sentencia recaída en el Exp. $\mathrm{N}^{\circ}$ 00045-2004-PI/TC. En la sentencia citada precedentemente, el Tribunal Constitucional, estableció los siguientes elementos: a) Determinación del tratamiento legislativo diferente: la intervención en la prohibición de discriminación, b) Determinación de la intensidad de la intervención en la igualdad, c) Determinación de la finalidad del tratamiento diferente (objetivo y fin), d) Examen de idoneidad, e) Examen de necesidad, y, f) Examen de proporcionalidad en sentido estricto o ponderación. 